\title{
Audio Transcript:
}

\section{Water-Soluble, Organic Fluorophores with Powerful Electrochemical and Photophysical Properties}

\author{
ACS Spring Meeting 2020 \\ Christian Fischer \\ (Sparr Research Group, University of Basel)
}

\section{Video link with subtitles: https://youtu.be/rgbcru3NZw4}

Title Slide:

Welcome to my presentation: "Water-Soluble, Organic Fluorophores with Powerful Electrochemical and Photophysical Properties", which is part of the virtual ACS Spring Meeting 2020. In the next couple of minutes, I will share with you the key results obtained during my $\mathrm{PhD}$ studies in the group of Prof. Christof Sparr at the University of Basel.

\section{Slide 2 (full-screen image):}

Small-molecule organic fluorophores are extremely fascinating and simply beautiful to look at.

\section{Slide 3 (full-screen image):}

Additionally, they are also very helpful in visualizing and supporting our understanding of the complex interplay of biomolecules. This is a fluorescence microscopy image with three distinct fluorophores: Fluorescein, rhodamine and DAPI.

\section{Slide 4:}

Century-long development led from these simple fluoresceins and rhodamines, quite complexly substituted organic fluorophores with distinct dye properties and diverse colors. This includes unsymmetrically substituted fluorophores such as ATTO 647 which is present as a racemate due to atropisomerism that occurs from the restricted rotation about the single bond. Interestingly, the distinct interactions of the two enantiomers in a biological environment are still unexplored today.

\section{Slide 5:}

Also current dye syntheses still rely on archetypical Baeyer condensation and Bernthsen-type reactions. These often require high reaction temperatures, an additional oxidation step or highly reactive reagents that limit the desired dye diversity. Also, the more modern heteroanthrone addition of organometallic reagents would require multiple steps to readjust and change the substitution pattern on the heterocyclic moiety. Therefore, a transformation of 
stable readily available starting materials accessing distinct fluorophores in a modular fashion under mild reaction conditions would be highly desirable.

\section{Slide 6 (full-screen image):}

Aside from bioimaging, small-molecule organic fluorophores are also becoming increasingly interesting for photocatalysis. The use of visible light and a photocatalyst enables the investigation of new reactions, which are not accessible under typical thermal conditions.

\section{Slide 7:}

Similar to fluorophores in bioimaging also in photocatalysis the excited-state lifetimes, the quantum yields of both fluorescence and intersystem crossing but also the molar absorption coefficient and absorption and emission wavelength are of great importance. Although in this short presentation I would like to focus for the most part only on the excited-and ground-state redox potentials as these are the most fundamental key to understanding the reactivities.

When a photocatalyst is irradiated by light, it can absorb energy from a photon and it excites an electron into a higher energy state. This provides an increased driving force for single electron transfer. Depending on the nature of the photocatalyst and its surroundings it can either act as an excited-state reductant, where an electron is released from the photocatalyst to the substrate or an oxidant. Or in another scenario, an electron is taken-up from the substrate or the reductant, and where the photocatalyst then acts as an excited-state oxidant. Subsequently, thermal re-reduction or re-oxidation then gives back the initial photocatalyst. This enables further reactions and eventually product formation. It is, however, very important that in both steps the half-reaction potential of the photocatalyst matches the half-reaction potential of the substrate to prevent side reactions or unproductive reactions from occurring. Therefore, we require access to various photocatalysts with quite nuanced redox properties.

\section{Slide 8:}

Today, most commonly used photocatalysts are these polybipyridyl based transition-metal complexes. Their excited-state reduction potential, shown here along this arrow, can be modulated by changing the ligand scaffold. This is not really possible for organic fluorophores but recently Nicewicz and DiRocco introduced electron-rich methoxy groups to lower the excited-state reduction potential of the highly oxidative Fukuzumi catalyst (MesMeAcr). However, as you can see, there is still room for improvement, as these organophotocatalysts do not come close to the powerful, yet expensive transition-metal complexes. Furthermore, current advances in photocatalysis with exciting applications in biology would benefit from the increased water-solubility of these acridinium derivatives. Similar to fluorophores used for imaging, we could also benefit here from a modular dye assembly strategy that accesses diverse substitution patterns with distinct redox properties over a broader range. 


\section{Slide 9:}

In a prototypical heterocyclic fluorophore, we would like to modulate five parts. This would be the heteroatom, in some cases substitution on the heteroatom, but also substituents on the $A$, $B$ and $C$ ring.

Arguably, in organic synthesis, we have good accessibility to various substituted arenes and coupling reactions to functionalize arenes and bridge the $A$ and $C$ ring via a heteroatom. However, we require improved methods for the formation of the $B$ ring under mild reaction conditions and enable various and diverse substitution.

Metalation would allow activation for a nucleophilic addition to carboxylic acid esters. Esters are ubiquitous and stable reagents, allowing for great diversity at this position and by choosing different metalation strategies the substitution pattern can be varied.

\section{Slide 10:}

By starting from dibromides, we could aim for a double halogen-metal exchange. But in the presence of directing groups such as these methoxys, this could enable a double directed ortho-metalation. Or of course, the combination of both strategies would allow for even further heterocyclic fluorophore derivatives after the addition to esters. With these strategies, we aimed at investigating a modular synthesis of xanthylium, SiR and acridinium fluorophores.

\section{Slide 11:}

Our starting point was built by the direct ester to arene transformation, where we used 1,5bifunctional organometallic reagents to allow for a double nucleophilic addition on esters to form cyclohexadienolate intermediates that are prone undergo a spontaneous 1,4-elimination to form substituted benzenes, anthracenes and different linear and nonlinear acenes. Together with my colleague Achim Link we found a high yielding method, which could form privileged scaffolds without transition-metals, yet use highly available starting materials under mild reaction conditions with a broad substrate scope. This rendered a reaction that later even high school students were able to successfully perform. This motivated us to introduce a heteroatom into the 1,5-bifunctional organometallic reagent and investigate the formation of heterocyclic fluorophores. However, the 1,4-elimination will no longer be possible with a heteroatom in the backbone, so we aimed for an acid promoted dehydration instead.

\section{Slide 12:}

The desired 1,5-bifunctional organometallic reagent to transform esters into xanthylium salts was synthesized in three steps. This involved a Cu-catalyzed Ullmann coupling that established the biaryl ether bridge, followed by a double bromination that proceeded with very high regioselectivity and a double halogen-metal exchange with magnesium. The reagent was directly added to methyl benzoate. After confirming the double addition and formation of the intermediary alkoxide, treatment with hydrobromic acid induced the dehydration. This is one of the most beautiful reactions l've ever seen, forming the rosamine bromide salt in its very 
intensive bright colors. This makes especially column chromatography very enjoyable as you can see here irradiated under $366 \mathrm{~nm}$ UV light.

\section{Slide 13:}

Aside from methyl benzoate we transformed electron-rich, electron-poor and sterically hindered, but also alkyl and alkenyl esters in good yield.

\section{Slide 14:}

This encouraged us to further investigate modern silicon-containing SiR dyes, which are especially useful in fluorescence microscopy due to their far-red emission and good water solubility. As the pioneering synthesis is step-intensive and of rather low robustness, we aimed to improve the accessibility of those dyes. A halogen-metal exchange and addition to dimethyldichlorosilane established the silicon bridge, followed by a beautifully regioselective double bromination that allowed the synthesis of the precursor in multigram scale. A double halogen-metal exchange gave access to the reagent, which remained stable for at least 60 days at room temperature under argon. The addition of this reagent to methyl benzoate still allowed for another beautiful reaction. After acidic work-up and purification leading to a nicely blue colored Si-rosamine.

\section{Slide 15:}

We investigated also a broad scope but especially interesting is that with this assembly strategy we can directly introduce various functional groups such as bromides or esters that allow bioconjugation and further functionalization without the need for additional steps or protecting groups. The protic aniline we installed by using an additional equivalent of the reagent and could confirm its structure by X-ray crystallography. The method proved to be highly robust, allowing the gram-scale synthesis of this derivative. Interestingly this aniline derivative has a very small fluorescence quantum yield, due to an intramolecular photoinduced electron transfer, which we could prevent by removing the electron density and installing electron-withdrawing groups, either via azide formation or simple acetylation. This allows for a 100-fold increase in fluorescence quantum yield, which makes this fluorescence enhancement strategy potentially useful for microscopy and bioassays.

\section{Slide 16:}

To apply the same fluorophore assembly strategy to acridinium salts, we coupled an aniline and iodoarene scaffold via Buchwald-Hartwig amination with the bromides already in place, followed by methylation and halogen-metal exchange. For photocatalysts, the two orthomethyl substituents of a mesityl moiety are indispensable as they shield parts of the acridinium from nucleophilic and radical attack. Therefore, it was particularly gratifying that we could access the highly sterically hindered mesityl derivative in $74 \%$ yield. 


\section{Slide 17:}

Changing now from double halogen-metal exchange to double directed ortho-metalation by simply using $n$-BuLi, allowed access to novel dimethoxy substituted acridinium salts, both $N$ methylated or $\mathrm{N}$-phenylated. A work-up with ammonium chloride instead of hydrobromic acid afforded access to the acridinol intermediate, but also the reaction with various esters could be investigated. We further introduced two or one dimethylamino groups, where we gained access to nonsymmetrically substituted acridinium salts. This allowed the formation of configurationally stable atropisomers that we separated by HPLC, equipped with a chiral stationary phase allowing future applications as enantiomerically enriched molecular probes.

\section{Slide 18:}

With the mixed metalation strategy, we obtained specifically unsymmetrically substituted acridinium salts: Methoxy dimethylamino or just one methoxy group or with a uniquely positioned dimethylamino group. We also examined the formation of xylyl instead of mesityl derivatives as the additional methyl group is often prone to undergo side reactions.

\section{Slide 19:}

Now let's go back to the beginning and look again at the excited-state reduction potential scale. Could we actually tune the dye properties by introducing this modular approach for the synthesis of diversely substituted heterocyclic fluorophores? The day we examined the properties of the diamino substituted acridinium salt for the first time was particularly exciting. Because, introducing dimethylamino groups influenced the dye properties dramatically. It showed a highly attenuated excited-state reduction potential compared to previously reported acridinium salts and the ground-state reduction potential was enhanced, matching the reduction properties of this frequently used heteroleptic iridium complex fairly well.

\section{Slide 20:}

Introducing 1,8-dimethoxy groups accessed further diversity for rather stronger photooxidants whereas the amino-substituted derivatives showed again more attenuated excited-state reduction potentials.

\section{Slide 21:}

The mixed metalation strategy then allowed modification over a broad range, accessing stronger and weaker organophoto-oxidants. One organophotocatalyst in particular, came close to both the excited- and ground-state reduction potentials of the trisbipyrazine ruthenium catalyst.

Nowadays, a lot of research groups develop and investigate very beautiful photocatalytic reactions by using the currently available catalysts. Our exciting results now pose the question if we could replace the expensive transition metal-based photocatalysts? 


\section{Slide 22:}

One of these examples is the ground-breaking photoredox-Nickel dual catalytic decarboxylative coupling described by Doyle and MacMillan using this heteroleptic iridium photocatalyst that allows very efficient transformations. Both the unsubstituted and tetramethoxy substituted acridinium salt fail in this transformation, presumably due to the mismatching excited-state and ground-state reduction potentials. However, the newly accessed diamino substituted acridinium salt that matches the reduction potentials allows catalysis and coupling product in $86 \%$ yield.

\section{Slide 23:}

In collaboration with Christoph Kerzig and Prof. Oliver Wenger, we examined the properties of the diaminoacridinium photocatalyst in more detail. Comparative DFT calculations of the Fukuzumi catalyst revealed fundamentally different HOMO-LUMO transitions. The introduction of dimethylamino groups evidently causes a change from the highly reactive charge-transfer character of the Fukuzumi catalyst to a less energetic $\pi-\pi^{*}$ character, seemingly more suited for complex multicomponent dual catalysis.

The unique properties of the diaminoacridinium catalyst were further underlined when we compared its reactivity to a large number of routinely used fluorophores and organic photocatalysts. Exclusively the diaminoacridinium photocatalyst led selectively to the desired $\mathrm{C}-\mathrm{C}$ coupling product. In contrast, for example, eosin $\mathrm{Y}$ led via a diverging triplet-triplet energy transfer pathway selectively to the $O$-aryl ester.

\section{Slide 24:}

The same iridium catalyst used for the decarboxylative C-C coupling could also successfully be replaced in a decarboxylative fluorination. The diaminoacridinium photocatalyst withstands effectively, possible catalyst degradation from the strongly electrophilic fluorinating reagent selectfluor.

\section{Slide 25:}

In another example, Yoon and co-workers investigated an oxidative $3+2$ cycloaddition to form dihydrobenzofurans by using this trisbipyrazine ruthenium catalyst. For this reaction, the diaminoacridinium photocatalyst was less suited but out of the mixed-metalation series we found a useful organophotocatalyst that allows for effective product formation.

\section{Slide 26:}

With this, I would like to conclude my talk. I hope I was able to convince you that the formation of 1,5-bifunctional organometallic reagents improves the diversity of currently accessible heterocyclic fluorophores by using the high availability and stability of esters, allowing for a milder assembly than ever before. Additionally, directed ortho-metalation and mixed 
metalation strategies can readily be applied to modulate the substituents on the heterocyclic fluorophores. Shown here with the focus to create acridinium dyes with diverse photophysical and electrochemical properties that are capable to be used as organophotocatalysts that can replace expensive and rare transition-metal based fluorophores. With the help of Solvias some of these powerful diaminoacridinium photocatalysts will soon be available through Strem Chemicals, Inc. and I hope to see many new applications and reactions evolving out of this.

\section{Acknowledgments (Slide 27):}

First and foremost, I would like to thank my PhD advisor, Prof. Christof Sparr, for his support and for letting me tackle such contemporary projects. I am particularly grateful to the collaboration and countless discussions with Prof. Oliver Wenger, Christoph Kerzig, Achim Link and Bouthayna Zilate. I am also thankful to Solvias AG for supporting our endeavor to commercialize our photocatalysts. I thank all the members of the Sparr research group for their support and friendship. I appreciated the invaluable support of Daniel Häussinger and Thomas Müntener in NMR spectroscopy and Markus Neuburger in X-ray crystallography.

Last but not least, I thank the University of Basel for funding me during my PhD studies and the Swiss National Science Foundation for an Early Postdoc Mobility Fellowship supporting my stay in the Trauner research group here at New York University.

Thank you for your attention and please reach out to us if you have any questions or comments. You can find our contact information on the last slide or the end of the audio transcript. Thank you very much.

\section{Christian Fischer}

christianfischer@nyu.edu,Twitter: @ChemFisch

\section{Christof Sparr}

christof.sparr@unibas.ch, http://sparr.chemie.unibas.ch/,

Twitter:@christof_sparr 